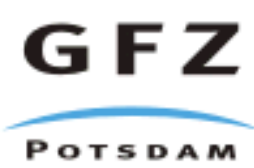

Originally published as:

Jahn, S., Madden, P. A. (2007): Modeling Earth materials from crustal to lower mantle conditions: A transferable set of interaction potentials for the CMAS system. - Physics of the Earth and Planetary Interiors, 162, 1-2, 129-139

DOI: 10.1016/j.pepi.2007.04.002 


\section{Accepted Manuscript}

Title: Modeling Earth materials from crustal to lower mantle conditions: A transferable set of interaction potentials for the CMAS system

Authors: Sandro Jahn, Paul A. Madden

PII:

S0031-9201(07)00062-3

DOI: doi:10.1016/j.pepi.2007.04.002

Reference: PEPI 4807

To appear in: Physics of the Earth and Planetary Interiors

Received date: 20-10-2006

Revised date:

Accepted date:

Please cite this article as: Jahn, S., Madden, P.A., Modeling Earth materials from crustal to lower mantle conditions: A transferable set of interaction potentials for the CMAS system, Physics of the Earth and Planetary Interiors (2007), doi:10.1016/j.pepi.2007.04.002

This is a PDF file of an unedited manuscript that has been accepted for publication. As a service to our customers we are providing this early version of the manuscript. The manuscript will undergo copyediting, typesetting, and review of the resulting proof before it is published in its final form. Please note that during the production process errors may be discovered which could affect the content, and all legal disclaimers that apply to the journal pertain. 


\title{
Modeling Earth materials from crustal to lower mantle conditions: A transferable set of interaction potentials for the CMAS system
}

\author{
Sandro Jahn* \\ GeoForschungsZentrum Potsdam, Department 4, Telegrafenberg, 14473 Potsdam, \\ Germany \\ Paul A. Madden \\ Chemistry Department, University of Edinburgh, Edinburgh EH9 3JJ, U.K.
}

\begin{abstract}
A fully flexible ionic interaction model (Aspherical Ion Model, AIM) is presented and applied to various relevant minerals and melts of the Ca-Mg-Al-Si-O (CMAS) system. The model reproduces accurately lattice parameters and thermoelastic properties of minerals in a wide range of compositions. Furthermore, its transferability range extends from the pressure and temperature conditions of the Earth's crust to the lower mantle. Thus, the AIM extends greatly the applicability of classical models in nanoscale computer simulations of Earth materials. The increased transferability compared to other interatomic potentials and the much more efficient calculation of interaction energies compared to first principles electronic structure calculations will allow larger scale simulations. The potential applications of the AIM to new fields of atomistic modeling in Earth sciences is discussed.
\end{abstract}

Key words: Molecular dynamics simulation; Equation of state; Phase transformation; Mantle transition zone; Lower mantle; Melt properties

\section{Introduction.}

Computer modeling has become a powerful tool to characterize the physical properties of geomaterials and to study geological processes on the nanoscale.

\footnotetext{
* Corresponding author.

Email address: jahn@gfz-potsdam.de (Sandro Jahn).
} 
Through direct access to the atomic structure and the respective atomic motions a fundamental atomistic understanding of macroscopic observations may be achieved. Furthermore, simulations allow to make predictions for physical and chemical properties of materials that are difficult to obtain experimentally, e.g. under extreme conditions of pressure and temperature.

The impact of atomistic modeling on current research in Earth Sciences is constrained mainly by two factors, the accuracy of the model and the accessible length and time scales. Due to the considerable increase in computing power in recent years, the usage of very accurate quantum-chemical methods to calculate structural and dynamic properties of complex geomaterials has become feasible. Electronic structure methods in the framework of density-functional theory (DFT) provide a means of modeling accurately a wide range of structures and chemical compositions from first principles, i.e. without specific assumptions for the atomic interaction in a specific structure. Approximations have to be made for the exchange-correlation functional. Although being accurate and transferable, DFT is currently limited to systems with a few hundred atoms and time scales of a few picoseconds. In many cases this is sufficient to study, e.g. the relative phase stability of different crystal phases as a function of pressure, $P$, and temperature, $T$ (Li et al., 2006a), the equations of state and the elastic constants of minerals (Li et al., 2006b), or crystal defect calculations (Zhang and Oganov, 2006).

For larger scale problems (hundreds to millions of atoms, hundreds of picoseconds to nanoseconds), required e.g. for modeling of viscous melts, interfaces or grain boundaries, classical interatomic potentials are usually employed. In this case, the atomic interactions are described by a more or less complex analytical function. Its parameters are optimized either by reference to experimental data (Matsui, 1999, 2000; Blanchard et al., 2005) or higher level theoretical methods (Tsuneyuki et al., 1988; van Beest et al., 1990; Tangney and Scandolo, 2002). Simple pairwise additive potentials may be parametrized to reproduce certain properties of a specific material, but they fail to describe non-central forces that lead, e.g. to the Cauchy violation in the elastic constants of cubic crystals $\left(c_{12} \neq c_{44}\right)$. Since the electronic structure especially of the oxygen ion, $\mathrm{O}^{2-}$, depends strongly on the neighboring ions, many body effects cannot be neglected in oxides and silicates.

Recently, a considerable progress towards more realistic modeling of geomaterials has been achieved by developing breathing shell models (BSMs) for $\mathrm{MgSiO}_{3}$ (Matsui, 2000), $\mathrm{Mg}_{2} \mathrm{SiO}_{4}$ (Matsui, 1999) and $\mathrm{Mg}$ spinels (Blanchard et al., 2005). These models take into account the ionic polarizability and shape deformations of the oxide ions. The respective potential parameters are usually optimized empirically, i.e. the simulated system is required to reproduce a number of experimental data. At this level, good agreement between model and experiment has been achieved for cell parameters and thermoelastic prop- 
erties. However, transferability is still rather limited since, e.g., different potential parameters are needed for modeling $\mathrm{MgSiO}_{3}$ and $\mathrm{Mg}_{2} \mathrm{SiO}_{4}$ (Matsui, 2000). The deficiencies of those interaction models may be caused by the model itself being too simple, by the way the potential parameters are determined, or both.

It would therefore be desirable to develop interaction potentials in a more systematic way. A method of constructing an aspherical ion model (AIM) for ionic materials from first principles has been described recently (Aguado et al., 2003a; Madden et al., 2006). Using similar ideas as the BSM, the AIM contains additional variables that represent the instantaneous changes of the electronic charge distribution. The analytical form of the AIM is derived from physical considerations and includes explicitly all contributions of the ionic interactions assumed to be relevant. This allows a well controlled optimization of the model parameters by reference to first principles DFT calculations only. Since no empirical data are used for model optimization, the comparison to experimental data provides a strong test for the predictive power of the model.

So far, AIMs have been developed for and successfully applied to simple halides and oxides (Aguado et al., 2002; Wilson et al., 2004). For oxides, they have been shown to be transferable in a wide range of pressure and temperature (Aguado and Madden, 2004), different chemical compositions (Aguado et al., 2003b; Aguado and Madden, 2003), and between different coordination environments, including crystalline structures, surfaces and melts (Jahn et al., 2004, 2006). In this paper, we explore for the first time the applicability of the AIM to more complex oxides and silicates. We choose the CMAS (CaO-MgO$\mathrm{Al}_{2} \mathrm{O}_{3}-\mathrm{SiO}_{2}$ ) model system, that contains major crustal and mantle minerals, e.g. quartz, forsterite, anorthite, pyroxenes, garnets, spinels, perovskite and post-perovskite structures. Further, the CMAS system may be used as a model for a dry basaltic melt. The inclusion of $\mathrm{H}_{2} \mathrm{O}$ and Fe-cations will be subject to future studies. Here, we will assess the ability of the model to reproduce quantitatively physical properties of the respective materials in a wide range of pressures and temperatures. Perspectively, such systematically constructed interaction models with a high degree of transferability may provide unprecedented insight into nanoscale structures and processes in geomaterials. This includes studies of the relation between atomic structure and transport properties of melts, melting and crystallization processes, atomic structure and transport in grain boundaries, and others.

\section{Interaction model.}

A detailed description of the AIM and its parametrization using first principles electronic structure calculations has been given elsewhere (Aguado et al., 
2003a; Madden et al., 2006). The present model is an extension of that recently published for $\mathrm{Al}_{2} \mathrm{O}_{3}$ (Jahn et al., 2006) to the CMAS system. For completeness, the analytical expressions of the ionic interactions are presented here again, together with the new set of potential parameters (Table 1).

The AIM potential is based on the classical theory of intermolecular forces (see e.g. Stone (1996)) and consists of a sum of four components: the pairwiseadditive Coulomb and dispersion interactions, a polarizable part and shortrange repulsion terms. The Coulomb term represents the charge-charge interaction between ions $i$ and $j$ separated with a distance $r_{i j}$

$$
V^{q q}=\sum_{i \leq j} \frac{q^{i} q^{j}}{r^{i j}}
$$

with $q^{i}$ being the formal charge on ion $i\left(q^{O}=-2, q^{M g}=q^{C a}=+2, q^{A l}=+3\right.$ and $\left.q^{S i}=+4\right)$. Dispersion effects are represented by dipole-dipole and dipolequadrupole terms

$$
V^{d i s p}=-\sum_{i \leq j}\left[1-f_{6}^{i j}\left(r^{i j}\right)\right] \frac{C_{6}^{i j}}{\left(r^{i j}\right)^{6}}+\left[1-f_{8}^{i j}\left(r^{i j}\right)\right] \frac{C_{8}^{i j}}{\left(r^{i j}\right)^{8}}
$$

where $C_{6}^{i j}$ and $C_{8}^{i j}$ are the dipole-dipole and dipole-quadrupole dispersion coefficients respectively, and $f_{n}^{i j}$ are Tang-Toennies dispersion damping functions (Tang and Toennies, 1984), which describe short-range corrections to the asymptotic dispersion term:

$$
f_{n}^{i j}\left(r^{i j}\right)=c_{n}^{i j} e^{-b_{n}^{i j} r^{i j}} \sum_{k=0}^{k_{\max }} \frac{\left(b_{n}^{i j} r^{i j}\right)^{k}}{k !}
$$

For the dispersion interactions we set $c_{6}^{i j}=c_{8}^{i j}=1, b_{6}^{i j}=b_{8}^{i j}$ and $k_{\max }=4$.

Polarization effects are considered up to the quadrupolar level (Wilson et al., 1996b). Since the ionic polarizabilities of light cations are usually much smaller than those of the anions (Heaton et al., 2006), only oxygen ions are regarded as being polarizable. Further, the oxygen polarizabilities are approximated by constants. A more elaborate treatment of polarizability would increase the complexity of the potential considerably, but may be unavoidable in future models (see discussion in section 4). The polarization part of the potential used here reads

$$
V^{p o l}=\sum_{i, j \in O}\left(\left(q^{i} \mu_{\alpha}^{j}-q^{j} \mu_{\alpha}^{i}\right) T_{\alpha}^{(1)}+\left(\frac{q^{i} \theta_{\alpha \beta}^{j}}{3}+\frac{\theta_{\alpha \beta}^{i} q^{j}}{3}-\mu_{\alpha}^{i} \mu_{\beta}^{j}\right) T_{\alpha \beta}^{(2)}\right.
$$




$$
\begin{aligned}
& \left.\left.+\left(\frac{\mu_{\alpha}^{i} \theta_{\beta \gamma}^{j}}{3}+\frac{\theta_{\alpha \beta}^{i} \mu_{\gamma}^{j}}{3}\right) T_{\alpha \beta \gamma}^{(3)}+\frac{\theta_{\alpha \beta}^{i} \theta_{\gamma \delta}^{j}}{9} T_{\alpha \beta \gamma \delta}^{(4)}\right)\right) \\
& +\sum_{i \in O, j \in C a, M g, A l, S i}\left(q^{j} \mu_{\alpha}^{i}\left[1-f_{D}^{i j}\left(r^{i j}\right)\right] T_{\alpha}^{(1)}+\frac{\theta_{\alpha \beta}^{i} q^{j}}{3}\left[1-f_{Q}^{i j}\left(r^{i j}\right)\right] T_{\alpha \beta}^{(2)}\right) \\
& +\sum_{i \in O}\left(\frac{1}{2 \alpha}\left|\vec{\mu}^{i}\right|^{2}+\frac{1}{6 C} \theta_{\alpha \beta}^{i} \theta_{\alpha \beta}^{i}\right)
\end{aligned}
$$

$\alpha$ and $C$ are the dipole and quadrupole polarizabilities of the anion, respectively. $T_{\alpha \beta \gamma \delta}=\nabla_{\alpha} \nabla_{\beta} \nabla_{\gamma} \nabla_{\delta} \ldots \frac{1}{r^{i j}}$ are the multipole interaction tensors (Stone, 1996). $\mu_{\alpha}^{i}(\alpha=x, y, z)$ are the Cartesian coordinates of the induced dipole on ion $i, \theta_{\alpha \beta}^{i}(\alpha, \beta=x, y, z)$ are the respective components of the quadrupole tensor. Summation over repeated indices is implied. For the short-range damping of the charge-dipole and charge-quadrupole cation-anion asymptotic functions again Tang-Toennies damping functions (Tang and Toennies, 1984) were used with $k_{\max }=4$ for the dipole $\left(f_{D}^{i j}\right)$ and $k_{\max }=6$ for the quadrupole $\left(f_{Q}^{i j}\right)$ damping functions. While the parameters $b_{D}^{i j}$ and $b_{Q}^{i j}$ determine the range at which the overlap of the charge densities affects the induced multipoles, the parameters $c_{D}^{i j}$ and $c_{Q}^{i j}$ determine the strength of the ion response to this effect.

For the short range repulsive terms of the potential deformable oxygen anions and rigid cations are considered. The repulsion between the small cations is assumed to be adequately modeled by the Coulombic term. The shape deformations are taken as relatively insignificant for the anion-anion repulsions, which are therefore represented by simple Born-Mayer exponentials, but they are substantial in the shell of nearest neighbors, i.e. for the anion-cation repulsion. The expression used here for the short range repulsion is given by

$$
\begin{array}{r}
V^{r e p}=\sum_{i \in O, j \in C a, M g, A l, S i}\left[A^{i j} e^{-a^{i j} \rho^{i j}}+B^{i j} e^{-b^{i j} \rho^{i j}}+\right. \\
\left.C^{i j} e^{-c^{i j} r^{i j}}\right]+\sum_{i, j \in O} A^{O O} e^{-a^{O O} r^{i j}}+ \\
\sum_{i \in O}\left[D\left(e^{\beta \delta \sigma^{i}}+e^{-\beta \delta \sigma^{i}}\right)+\left(e^{\zeta^{2}\left|\nu^{i}\right|^{2}}-1\right)+\right. \\
\left.\left(e^{\eta^{2}\left|\kappa^{i}\right|^{2}}-1\right)\right],
\end{array}
$$

where

$$
\rho^{i j}=r^{i j}-\delta \sigma^{i}-S_{\alpha}^{(1)} \nu_{\alpha}^{i}-S_{\alpha \beta}^{(2)} \kappa_{\alpha \beta}^{i},
$$

and summation of repeated indices is implied. The variable $\delta \sigma^{i}$ characterizes the deviation of the radius of oxide anion $i$ from its default value, $\left\{\nu_{\alpha}^{i}\right\}$ are a set of three variables describing the Cartesian components of a dipolar 
distortion of the ion, and $\left\{\kappa_{\alpha \beta}^{i}\right\}$ are a set of five independent variables describing the corresponding quadrupolar shape distortions. In Eqn. 5, $|\kappa|^{2}=$ $\kappa_{x x}^{2}+\kappa_{y y}^{2}+\kappa_{z z}^{2}+2\left(\kappa_{x y}^{2}+\kappa_{x z}^{2}+\kappa_{y z}^{2}\right)$ and $S_{\alpha}^{(1)}=r_{\alpha}^{i j} / r^{i j}$ and $S_{\alpha \beta}^{(2)}=3 r_{\alpha}^{i j} r_{\beta}^{i j} / r^{i j^{2}}-\delta_{\alpha \beta}$ are interaction tensors. The last summations include the self-energy terms, representing the energy required to deform the anion charge density, with $\beta, \zeta$ and $\eta$ as effective force constants. The extent of each ion's distortion is determined at each molecular dynamics (MD) time-step by energy minimization. Especially for the high pressure phases, the introduction of an additional 'rigid' Born-Mayer-type term in the anion-cation repulsion interaction has proven useful. This extra exponential function $\left(C^{-+} e^{-c^{-+} r^{i j}}\right.$ in Eqn. 5) accounts for the hard core of the anion.

The parameters of the AIM potentials are optimized by reference to first principles DFT calculations. Atomic configurations of typically 60 to 120 ions representing different minerals and melts of the CMAS system are chosen as a training set. The configurations are taken from short MD simulations at different conditions of $P$ and $T$ or created by setting up supercells of crystal structures with the ions randomly displaced from their lattice sites. In total, the training set consists of 19 configurations, including high and low pressure polymorphs of $\mathrm{Al}_{2} \mathrm{O}_{3}(4), \mathrm{MgO}(3), \mathrm{SiO}_{2}(3), \mathrm{MgAl}_{2} \mathrm{O}_{4}(3), \mathrm{MgSiO}_{3}(2), \mathrm{CaO}$ (1), $\mathrm{CaAl}_{2} \mathrm{Si}_{2} \mathrm{O}_{8}(1)$, and two $\mathrm{Al}_{2} \mathrm{O}_{3}$ melt configurations.

For each configuration, single point energy DFT calculations are performed using the plane-wave code CASTEP (Payne et al., 1992). In all calculations, Vanderbilt ultrasoft potentials (Vanderbilt, 1990) and a planewave cutoff of $653 \mathrm{eV}$ are used. The exchange-correlation functional is treated in the local density approximation (LDA). From the DFT calculations, the force vector acting on each ion and the stress tensor of the simulation cell are obtained. After transforming the Kohn-Sham orbitals into sets of maximally localized Wannier functions (Marzari and Vanderbilt, 1997), ab initio dipoles and quadrupoles are calculated (Aguado et al., 2003a).

Subsequently, the AIM parameters are obtained by fitting classical forces, stresses and multipoles to the corresponding $a b$ initio data. In a first step, the polarizable part of the model (Eq. 4) is optimized by fitting the multipoles only. In the second part of the fitting procedure, the short-range repulsive terms and the deformation self-energy parameters (Eq. 5) are optimized by fitting the forces and stress tensors. The parameters of the dispersion interactions remain fixed to the values of the earlier alumina potential since dispersion is not well represented by standard DFT (see Jahn et al. (2006) for a discussion). The resulting potential parameters are listed in Table 1. 


\section{Transferability tests.}

The quality of the new interaction model may be assessed by comparing model predictions to experimental or to electronic structure data. Since no experimental data was used in the optimization of the model parameters, a strong test for the quality of the model is whether it is able to reproduce experimental data of physical properties for different minerals or melts in the CMAS system in a wide range of pressures and temperatures. The set of test cases that will be presented in the following was chosen to demonstrate the applicability of the model to minerals and melts abundant in the Earth's crust down to those of the core-mantle boundary. Most of the minerals chosen here are not included in the training set. Thus the results provide a true transferability check.

\subsection{Lattice parameters and elastic constants.}

First, lattice parameters and elastic constants of various minerals at ambient conditions are considered. Whereas the lattice constants represent the average structure, the elastic constants are indicative of the force constants between the ions. For each of the following structures, the cell and atomic parameters were optimized by minimizing the forces between the atoms and the stress tensor of the simulation cell using standard minimization algorithms (Press et al., 1989). For the calculation of the elastic constants, the optimized simulation cell is strained in different directions by a small amount (typically a fraction of a percent) and the resulting stress tensor is recorded after relaxation of the atomic positions. Repeating this procedure for several magnitudes of positive or negative strain, the linear relationship between strain and stress is used to obtain the elastic constants.

To demonstrate transferability, the AIM predictions and experimental data are compared for polymorphic phases of the $\mathrm{Mg}_{2} \mathrm{SiO}_{4}$ stoichiometry (Tables 2,3 and 4), for end member garnets (Table 5), and for low pressure phases of the $\mathrm{Al}_{2} \mathrm{O}_{3}-\mathrm{SiO}_{2}$ system (Table 6). For $\mathrm{Mg}_{2} \mathrm{SiO}_{4}$, results from other recent studies using interatomic potentials (Matsui, 1999; Blanchard et al., 2005) are included, as well.

The agreement between AIM predictions and experiment for most of the examples presented here is of the order of one percent in the lattice parameters and ten percent in the elastic constants, or better. This precision is similar to that obtained in electronic structure calculations. The systematic underestimation of the lattice constants by the AIM is due to the use of the LDA in the reference electronic structure calculations. Larger deviations from exper- 
imental results are observed in less dense packed structures, such as quartz $\left(\alpha-\mathrm{SiO}_{2}\right)$. This is probably related to the increased covalent character of the Si-O bonds, which may set a limit to the transferability of the AIM within the approximations applied here (e.g. constant anion polarizability and deformability).

Although the AIM is applicable to a wider range of materials, its results compare favorably with other, more dedicated interatomic potentials. Only Matsui's potential (Matsui, 1999) that was specifically parametrized for $\mathrm{Mg}_{2} \mathrm{SiO}_{4}$ polymorphs, performs slightly better in terms of lattice and elastic constants than the AIM. However, no application of this set of parameters to other stoichiometries or chemical compositions has been published. Blanchard et al. (2005) optimized their potential for spinel-type structures. In their paper, they already discuss the transferability problems to the other polymorphs of $\mathrm{Mg}_{2} \mathrm{SiO}_{4}$, which can be seen in some larger deviations from the experimental data in the elastic constants of forsterite and wadsleyite.

\subsection{Equations of state of forsterite and ringwoodite.}

The lattice parameters and elastic constants presented above refer to ambient pressure and low temperature conditions. Now, the transferability of the AIM to high pressures and temperatures is demonstrated by calculating the equations of state of two $\mathrm{Mg}_{2} \mathrm{SiO}_{4}$ polymorphs, forsterite and ringwoodite. Both minerals are end members of olivine and spinel compounds that are considered the main constituents of the upper mantle and the transition zone. The respective conditions in the Earth reach up to pressures of about $20 \mathrm{GPa}$ and temperatures of about $2000 \mathrm{~K}$. The results in this subsection were obtained from molecular dynamics simulations using a Nose-Hoover thermostat coupled to a barostat (Martyna et al., 1994). For forsterite, the simulation cell contained 672 ions. Simulations with 2520 ions were performed to confirm convergence of the results with regard to finite size effects. The simulation cell of ringwoodite contained 448 ions. After equilibration at each pressure and temperature, the volume was obtained from averaging over $10 \mathrm{ps}$ simulations with a time step of $1 f s$.

Forsterite, $\alpha-\mathrm{Mg}_{2} \mathrm{SiO}_{4}$, is the magnesium end member of olivine that is considered the principal mineral of the upper mantle. In Fig. 1 the volume compression of forsterite at $T=300 \mathrm{~K}$ is shown as a function of pressure. Over the whole pressure range, the molar volume predicted by the AIM is in excellent agreement with experimental data (Downs et al., 1996) with maximum deviations of less than one percent. The thermal expansion of forsterite at $P=0$ is shown in Fig. 2. Again, the agreement of AIM predictions with experimental data (Bouhifd et al., 1996) is very good over a wide temperature range. 
Ringwoodite, $\gamma-\mathrm{Mg}_{2} \mathrm{SiO}_{4}$, is a high pressure polymorph of forsterite with a spinel crystal structure. Its stability field is expected to be in the lower part of the transition zone. Experimental studies of the equation of state therefore concentrate on compression studies at high pressure up to $30 \mathrm{GPa}$ (Meng et al., 1994) and more recently to relevant high pressure and high temperature conditions, i.e. temperatures up to $2000 \mathrm{~K}$ and pressures from 15 to $24 \mathrm{GPa}$ (Katsura et al., 2004). In Table 4 it was shown that the lattice constant for ringwoodite predicted by the model is about one percent lower than the experimental data. This accumulates to a systematic three percent deviation in the molar volume. For a better comparison, the volume change with pressure and temperature is therefore compared relative to the respective volume at ambient conditions (Fig. 3). The transferability to transition zone conditions is fairly good considering the spread of the experimental data. It seems that at high pressures, the model predicts slightly smaller compressibility than the experiment.

\subsection{Low to high pressure phase transformation in clinoenstatite.}

Enstatite, $\mathrm{MgSiO}_{3}$, is an end-member of silicate pyroxene, a major constituent mineral of the upper mantle. The P-T phase diagram of $\mathrm{MgSiO}_{3}$ suggests that there are five thermodynamically stable polymorphs with pyroxene structure, three monoclinic and two orthorhombic phases (Presnall, 1995). At low temperature $(T$ below $\sim 800 K)$, low temperature $(\mathrm{LT})$ clinoenstatite (space group $\mathrm{P} 2_{1} / \mathrm{c}$ ) transforms to the non-quenchable high-pressure (HP) clinoenstatite phase (space group $\mathrm{C} 2 / \mathrm{c}$ ) at pressures between 5 and $7 \mathrm{GPa}$. The knowledge of the mechanism and kinetics of phase transformations is important for understanding dynamical processes in the Earth's interior. The LT-HP transition has been the subject of both experimental studies (Angel et al., 1992) and computer simulations (Mendelssohn and Price, 1997). It has been shown to be a displacive phase transition that is accompanied by small rotations of the $\mathrm{SiO}_{4}$ tetrahedra. Here, we demonstrate that the AIM predicts the transition in the same pressure range as previous studies and that the transition is reversible in the time scale of the simulation.

Fig. 4 shows the evolution of the cell volume across the transition obtained from a molecular dynamics simulation at constant temperature $(T=300 \mathrm{~K})$ using the AIM potential. Starting with the LT phase at ambient conditions, the pressure was raised steadily with a rate of $0.5 \mathrm{GPa} / \mathrm{ps}$. The transition to the HP phase occurs at about $P=10 G P a$. The transition is accompanied by a sudden decrease in volume. On the reverse path, the pressure was decreased at the same rate and the transition happened at about $P=1 G P a$. With increasing temperature, the hysteresis of the transition pressure is decreased to $\triangle P \sim 1 G P a$ at $T=1000 \mathrm{~K}$. The mean transition pressure at $T=1000 \mathrm{~K}$ 
is about $5.5 \mathrm{GPa}$. These results are consistent with the experimental data, which bracket the transition between 5.3 and $\sim 8.0 \mathrm{GPa}$ at $\mathrm{T}=300 \mathrm{~K}$ (Angel et al., 1992), and previous simulations, where the transitions appears at $6 \mathrm{GPa}$ (Mendelssohn and Price, 1997).

\subsection{Lower mantle phases.}

After demonstrating transferability of the AIM between conditions of the crust and the upper mantle, this subsection is dedicated to lower mantle phases. Silicate perovskite, $(\mathrm{Mg}, \mathrm{Fe}) \mathrm{SiO}_{3}$, is considered the major constituent mineral of the lower mantle. The magnesium end member, $\mathrm{MgSiO}_{3}$ perovskite, has been subject to a large number of experimental and theoretical studies, also at $\mathrm{P} / \mathrm{T}$ conditions of the lower mantle (Fiquet et al., 2000; Wentzcovitch et al., 2004; Li and Zhang, 2005). The recently discovered post-perovskite phase (Murakami et al., 2004; Oganov and Ono, 2004), a very high pressure polymorph of $\mathrm{MgSiO}_{3}$, may be responsible for the $\mathrm{D}^{\prime \prime}$ seismic discontinuity and the seismic anisotropies in the $\mathrm{D}^{\prime \prime}$ layer.

In Table 7 , the lattice parameters and elastic constants of the perovskite and post-perovskite phases predicted by the AIM $(P=120 \mathrm{GPa}, \mathrm{T}=300 \mathrm{~K})$ are compared to recent results from electronic structure calculations $(P=$ $120 \mathrm{GPa}$ and $T=0 \mathrm{~K}$ ) (Iitaka et al., 2004). Experimental values for the elastic constants at those extreme conditions are still not available. The agreement is of the same order as for the mineral phases at ambient or low pressure presented above. The lattice parameters agree with the reference data within one percent, the elastic constants in most cases within $10 \%$. There are some larger deviations in $c_{13}$ and $c_{23}$ of both phases and $c_{66}$ of perovskite.

Good transferability is also obtained for two other important phases considered abundant in the lower mantle, the magnesium end member of $(\mathrm{Mg}, \mathrm{Fe}) \mathrm{O}$ magnesiow üstite and $\mathrm{CaSiO}_{3}$ perovskite. The lattice constant of $\mathrm{MgO}$ at $P=$ $100 \mathrm{GPa}$ is predicted to be $a=3.75 \AA$, which is in good agreement with DFT results by Oganov and Dorogokupets (2003) $(a=3.80 \AA)$. The slightly smaller value of the AIM lattice constant is probably caused by the use of LDA in the fitting, whereas the calculations in Oganov and Dorogokupets (2003) were performed within the generalized gradient approximation (GGA). Recent studies on $\mathrm{CaSiO}_{3}$ (Adams and Oganov, 2006; Li et al., 2006a) suggest that the ground state structure at low temperature is tetragonal or orthorhombic in wide pressure range. The present AIM predicts a tetragonal phase of $\mathrm{CaSiO}_{3}$ at $P=100 \mathrm{GPa}$ and $T=0$ with lattice constants of $a=3.25 \AA$ and $c=3.32 \AA$. This is consistent with DFT calculations of Adams and Oganov (2006), who predict $a=3.28 \AA$ and $c=3.34 \AA$ (using again GGA). 


\subsection{Melts}

One of the motivations for the construction of interatomic potentials is their usability in larger scale molecular dynamics simulations compared to respective $a b$ initio MD simulations. The former are especially needed to study nonideal solid state problems, such as defects, grain boundaries or solid-melt interfaces, and melts. The experimental study of such problems often proves extremely difficult, especially properties have to be measured in situ at high pressure and high temperature. For this reason, the knowledge of melt structures and melt properties under mantle conditions is yet very limited.

First applications of the AIM to melts were focussed on oxide melts. The model reproduces well the atomic structure and dynamics of $\mathrm{Al}_{2} \mathrm{O}_{3}-\mathrm{MgO}$ melts (Krishnan et al., 2005; Jahn and Madden, 2006; Pozdnyakova et al., 2007) and also yields viscosities in agreement with experiment (Jahn et al., 2006). At high pressure, structural changes in the melt lead to a considerably higher compressibility compared to the solid phase. This is illustrated in Fig. 5, where the $\mathrm{P}-\mathrm{V}$ curves of corundum and $\mathrm{Al}_{2} \mathrm{O}_{3}$ melt are shown and compared to experimental data. For corundum, the molar volumes predicted by the AIM are systematically smaller than the experimental data (Dubrovinsky et al., 1998; Grevel et al., 2000) by about $2 \%$, but the slope of the compression curve is in very good agreement with experiment. This is consistent with the results of the silicate minerals discussed above. The density of the melt at $T=2500 \mathrm{~K}$ and $P=0 G P a$ from the simulation is almost identical to recent experimental results (Glorieux et al., 1999). At high pressure, the melt density approaches the density of the crystal bearing in mind the thermal expansion of the crystal at high temperatures, which is almost $7 \%$ at $T=2300 \mathrm{~K}$ and $P=0 \mathrm{GPa}$ but slightly less at high pressure. Eventually, the melt may become denser than the solid, which would have severe consequences on the buoyancy behavior of melts in the Earth's interior. A more detailed discussion of these results and extensions of the AIM simulations to silicate melts will be given elsewhere.

\section{Discussion and conclusions.}

In the previous section it has been shown that the systematic approach of developing AIM-type interatomic potentials from first-principles electronic structure calculations may be extended to complex multicomponent ionic materials, such as oxides and silicates. With the new set of potentials, an accurate description of the structure and physical properties of geologically relevant minerals and melts of the CMAS system is obtained in a wide range of pressures and temperatures. To our knowledge, none of the existing interatomic potentials has been applied across such a wide range of materials and thermo- 
dynamic conditions.

In many of the transferability tests presented here, the accuracy of the AIM is comparable to results from electronic structure calculations. This suggests that the essential contributions to the atomic interactions are included in our classical model, at least in the range of applications discussed here. The transferability range of the AIM compared to other interatomic potentials seems to be greatly improved. The AIM is not intended to compete with electronic structure calculations, but it is merely intended as a model for materials that are dominated by ionic bonding including some degree of covalency. However, the AIM is able to predict reasonably well physical properties of different minerals, such as elastic constants and equations of state, the thermodynamic stability regions of high pressure mineral phases, and to provide a good description of melt structures and properties with considerably decreased computational cost. For example, a ten picosecond MD simulation with a time step of one femtosecond, 1000 atoms in the simulation cell, takes about 24 hours on a single modern CPU. A parallel version of the AIM code using Message Passing Interface (MPI) is now also available for larger scale simulations. This may open new fields of exploration for atomistic modeling of geological materials, e.g. structure of grain boundaries and their influence on properties of composites, or mineral-melt interfaces and study of element partitioning. Moreover, since the AIM is based on physical considerations about interatomic forces, the influence of the individual contributions to the interaction on the physical properties may be studied. For example, the stability of corundum $\left(\alpha-\mathrm{Al}_{2} \mathrm{O}_{3}\right)$ seems to depend crucially on the induction of a quadrupolar moment on the oxygen ion (Wilson et al., 1996a). Such electronic effects may also be responsible for the specific evolution of elastic properties with pressure and temperature. Most of these effects are largely unexplored.

The current version of the AIM already performs well in many cases. However, there is still need for improvement of the model or the way it is parametrized. Although the polarizable part of the model is optimized independently from the other terms, the effects of parameter correlations have to be studied in detail. Furthermore, the effects of the atomic environment on the ionic polarizabilities and deformations may have to be included explicitly. A case study for $\mathrm{MgO}$ (Aguado and Madden, 2004) has shown excellent transferability to very high pressures, but a more robust approach of including such effects still has to be developed. Recently, a new method to calculate polarizabilities of single ions in condensed phases from DFT calculations has been presented (Heaton et al., 2006). This approach may be used for implementing environmental effects into the AIM.

Other deviations of AIM predictions from experimental data are related to the underlying approximations of the DFT calculations. The use of LDA for the exchange-correlation functional in the DFT calculations leads to lattice 
constants that are systematically about $1 \%$ too small. This may effect the elastic constants. For the cubic crystals (ringwoodite, garnets) the smaller lattice constants seems to lead to a systematic overestimation of the elastic constants predicted by the AIM. This is consistent with the increase of the elastic constants with pressure (Sinogeikin and Bass, 2000). It is not trivial to improve systematically the accuracy of the DFT calculations. The use of the generalized gradient approximation (GGA) instead of LDA lead to better agreement in some of the lattice parameters, but caused melt densities much too low (Jahn et al., 2006). Another challenge for future AIM developments is the inclusion of further important ions, such as the alkali cations $\left(\mathrm{Na}^{+}, \mathrm{K}^{+}\right)$, $\mathrm{Fe}^{2+}$ and $\mathrm{Fe}^{3+}$, or molecular units $\left(\mathrm{OH}^{-}, \mathrm{NH}_{4}^{+}, \mathrm{CO}_{3}^{2-}\right)$.

Ultimately, the AIM may provide physical properties of complex materials (melts, solid solutions, etc.) as input parameters for large scale geophysical models. Often, these properties are not known since their experimental determination is not yet possible and computational demand for electronic structure calculations is still too large. On the other hand, interatomic potentials less advanced than the AIM may not have the predictive power to be transfered to the respective thermodynamic conditions of high pressure and temperature.

\section{Acknowledgments}

We thank F. Schilling and S. Speziale for helpful comments and discussions.

\section{References}

Adams, D. J., Oganov, A. R., 2006. Ab initio molecular dynamics study of $\mathrm{CaSiO}_{3}$ perovskite at P-T conditions of earth's lower mantle. Phys. Rev. B 73, 184106.

Aguado, A., Bernasconi, L., Jahn, S., Madden, P. A., 2003a. Multipoles and interaction potentials in ionic materials from planewave-DFT calculations. Faraday Discuss. 124, 171-184.

Aguado, A., Bernasconi, L., Madden, P. A., 2002. A transferable interatomic potential for $\mathrm{MgO}$ from ab initio molecular dynamics. Chem. Phys. Lett. $356,437-444$.

Aguado, A., Bernasconi, L., Madden, P. A., 2003b. Interionic potentials from ab initio molecular dynamics: The alkaline earth oxides $\mathrm{CaO}, \mathrm{SrO}$ and $\mathrm{BaO}$. J. Chem. Phys. 118, 5704-5717.

Aguado, A., Madden, P. A., 2003. Oxide potentials from ab initio molecular dynamics: An assessment of their transferability. J. Chem. Phys. 118, 57185728. 
Aguado, A., Madden, P. A., 2004. Fully transferable interatomic potentials for large-scale computer simulations of simple metal oxides: Application to MgO. Phys. Rev. B 70, 245103.

Angel, R. J., Chopelas, A., Ross, N. L., 1992. Stability of high-density clinoenstatite at upper-mantle pressures. Nature 358, 322-324.

Armbruster, T., Geiger, C. A., Lager, G. A., 1992. Single-crystal x-ray structure study of synthetic pyrope almandine garnets at 100 and $293 \mathrm{~K}$. Am. Mineral. 77, 512-521.

Bass, J. D., 1989. Elasticity of grossular and spessartite garnets by brillouin spectroscopy. J. Geophys. Res. 94, 7621-7628.

Blanchard, M., Wright, K., Gale, J. D., 2005. Atomistic simulation of $\mathrm{Mg}_{2} \mathrm{SiO}_{4}$ and $\mathrm{Mg}_{2} \mathrm{GeO}_{4}$ spinels: a new model. Phys. Chem. Minerals 32, 332-338.

Bouhifd, M. A., Andrault, D., Fiquet, G., Richet, P., 1996. Thermal expansion of forsterite up to the melting point. Geophys. Res. Lett. 23, 1143-1146.

Downs, R. T., Zha, C., Duffy, T. S., Finger, L. W., 1996. The equation of state of forsterite to $17.2 \mathrm{GPa}$ and effects of pressure media. Am. Mineral. $81,51-55$.

Dubrovinsky, L. S., Saxena, S. K., Lazor, P., 1998. High-pressure and hightemperature in situ x-ray diffraction study of iron and corundum to $68 \mathrm{GPa}$ using an internally heated diamond anvil cell. Phys. Chem. Minerals 25, 434-441.

Fiquet, G., Dewaele, A., Andrault, D., Kunz, M., le Bihan, T., 2000. Thermoelastic properties and crystal structure of $\mathrm{MgSiO}_{3}$ perovskite at lower mantle pressure and temperature conditions. Geophys. Res. Lett. 27, 21-24.

Fujino, K., Sasaki, S., Takeuchi, Y., Sadanaga, R., 1981. X-ray determination of electron distributions in forsterite, fayalite and tephroite. Acta Cryst. B $37,513-518$.

Glorieux, B., Millot, F., Rifflet, J.-C., Coutures, J.-P., 1999. Density of superheated and undercooled liquid alumina by a contactless method. Int. J. Thermophys. 20, 1085-1094.

Grevel, K.-D., Burchard, M., Faßhauer, D. W., 2000. Pressure-volumetemperature behavior of diaspore and corundum: An in situ x-ray diffraction study comparing different pressure media. J. Geophys. Res. 105, 2787727887.

Hearmon, R. F. S., 1979. Landolt-Börnstein Tables, III/11. Springer, Ch. The elastic constants of crystals and other anisotropic materials, pp. 1-154.

Heaton, R. J., Madden, P. A., Clark, S. J., Jahn, S., 2006. Condensed phase ionic polarizabilities from plane wave density functional theory calculations. J. Chem. Phys. 125, 144104.

Horiuchi, H., Sawamoto, H., 1981. $\beta-\mathrm{Mg}_{2} \mathrm{SiO}_{4}$ : single crystal x-ray diffraction study. Am. Mineral. 66, 568-575.

Iitaka, T., Hirose, K., Kawamura, K., Murakami, M., 2004. The elasticity of the $\mathrm{MgSiO}_{3}$ post-perovskite phase in the earth's lowermost mantle. Nature 430, 442-445.

Jahn, S., Madden, P. A., 2006. Structure and dynamics in liquid alumina: 
simulations with an ab initio interaction potential. J. Non-Cryst. Solids , submitted.

Jahn, S., Madden, P. A., Wilson, M., 2004. Dynamic simulation of the pressure-driven phase transformations in crystalline $\mathrm{Al}_{2} \mathrm{O}_{3}$. Phys. Rev. B 69, 020106(R).

Jahn, S., Madden, P. A., Wilson, M., 2006. Transferable interaction model for $\mathrm{Al}_{2} \mathrm{O}_{3}$. Phys. Rev. B 74, 024112.

Katsura, T., Yokoshi, S., Song, M., Kawabe, K., Tsujimura, T., Kubo, A., Ito, E., Tange, Y., Tomioka, N., Saito, K., Nozawa, A., Funakoshi, K., 2004. Thermal expansion of $\mathrm{Mg}_{2} \mathrm{SiO}_{4}$ ringwoodite at high pressures. J. Geophys. Res. 109, B12209.

Krishnan, S., Hennet, L., Jahn, S., Key, T. A., Saboungi, M.-L., Madden, P. A., Price, D. L., 2005. The structure of normal and supercooled liquid aluminum oxide. Chem. Mater. 17, 2662-2666.

LePage, Y., Calvert, L. D., Gabe, E. J., 1980. Parameter variation in low quartz between 94 and 298 K. J. Phys. Chem. Solids 41, 721-725.

Li, B., Zhang, J., 2005. Pressure and temperature dependence of elastic wave velocity of $\mathrm{MgSiO}_{3}$ perovskite and the composition of the lower mantle. Phys. Earth Planet. Int. 151, 143-154.

Li, L., Weidner, D. J., Brodholt, J., Alfe, D., Price, D. G., Caracas, R., Wentzcovitch, R., 2006a. Phase stability of $\mathrm{CaSiO}_{3}$ perovskite at high pressure and temperature: Insights from ab initio molecular dynamics. Phys. Earth Planet. Int. 155, 260-268.

Li, L., Weidner, D. J., Brodholt, J., Alfe, D., Price, G. D., Caracas, R., Wentzcovitch, R., 2006b. Elasticity of $\mathrm{CaSiO}_{3}$ perovskite at high pressure and high temperature. Phys. Earth Planet. Int. 155, 249-259.

Madden, P. A., Heaton, R., Aguado, A., Jahn, S., 2006. From first-principles to material properties. J. Mol. Struc. (Theochem) 771, 9-18.

Martyna, G. J., Tobias, D. J., Klein, M. L., 1994. Constant pressure molecular dynamics algorithms. J. Chem. Phys. 101, 4177-4189.

Marzari, N., Vanderbilt, D., 1997. Maximally localized generalized Wannier functions for composite energy bands. Phys. Rev. B 56 (20), 12847.

Matsui, M., 1999. Computer simulation of the $\mathrm{Mg}_{2} \mathrm{SiO}_{4}$ phases with application to the $410 \mathrm{~km}$ seismic discontinuity. Phys. Earth Planet. Int. 116, 9-18.

Matsui, M., 2000. Molecular dynamics simulation of $\mathrm{MgSiO}_{3}$ perovskite and the 660-km seismic discontinuity. Phys. Earth Planet. Int. 121, 77-84.

Mendelssohn, M. J., Price, G. D., 1997. Computer modelling of a pressure induced phase change in clinoenstatite pyroxenes. Phys. Chem. Minerals $25,55-62$.

Meng, Y., Fei, Y., Weidner, D. J., Gwanmesia, G. D., Hu, J., 1994. Hydrostatic compression of $\gamma-\mathrm{Mg}_{2} \mathrm{SiO}_{4}$ to mantle pressures and $700 \mathrm{~K}$ : Thermal equation of state and related thermoelastic properties. Phys. Chem. Minerals 21, 407412 .

Murakami, M., Hirose, K., Kawamura, K., Sata, N., Ohishi, Y., 2004. Post- 
perovskite phase transition in $\mathrm{MgSiO}_{3}$. Science 304, 855-858.

Newnham, R. E., de Haan, Y. M., 1962. Refinement of the alpha- $\mathrm{Al}_{2} \mathrm{O}_{3}, \mathrm{Ti}_{2} \mathrm{O}_{3}$, $\mathrm{V}_{2} \mathrm{O}_{3}$ and $\mathrm{Cr}_{2} \mathrm{O}_{3}$ structures. Z. Krist. 117, 235-237.

Novak, G. A., Gibbs, G. V., 1971. The crystal chemistry of the silicate garnets. Am. Mineral. 56, 791-825.

Oganov, A. R., Dorogokupets, P. I., 2003. All-electron and pseudopotential study of $\mathrm{MgO}$ : Equation of state, anharmonicity, and stability. Phys. Rev. B 67, 224110.

Oganov, A. R., Ono, S., 2004. Theoretical and experimental evidence for a post-perovskite phase of $\mathrm{MgSiO}_{3}$ in Earth's D" layer. Nature 430, 445-448.

O’Neill, B., Bass, J. D., Rossman, G. R., Geiger, C. A., Langer, K., 1991. Elastic properties of pyrope. Phys. Chem. Minerals 17, 617-621.

Payne, M. C., Teter, M. P., Allan, D. C., Arias, T. A., Joannopoulos, J. D., 1992. Iterative minimization techniques for ab initio total-energy calculations: molecular dynamics and conjugate gradients. Rev. Mod. Phys. 64, 1045-1097.

Pozdnyakova, I., Hennet, L., Brun, J.-F., Zanghi, D., Brassamin, S., Cristiglio, V., Price, D. L., Albergamo, F., Bytchkov, A., Jahn, S., Saboungi, M.-L., 2006. Longitudinal excitations in Mg-Al-O refractory oxide melts studied by inelastic x-ray scattering. J. Chem. Phys., in press.

Presnall, D. C., 1995. Mineral Physics and Crystallography, A Handbook of Physical Constants. Vol. 2 of AGU Reference Shelf. American Geophysical Union, Ch. Phase diagrams of Earth-forming minerals, pp. 248-268.

Press, W., Flannery, B., Teukolsky, S., Vetterling, W., 1989. Numerical Recipes. Cambridge University Press.

Sasaki, S., Prewitt, C. T., Sato, Y., Ito, E., 1982. Single-crystal x ray study of $\gamma \mathrm{Mg}_{2} \mathrm{SiO}_{4}$. J. Geophys. Res. 87, 7829-7832.

Sawamoto, H., Weidner, D. J., Sasaki, S., Kumazawa, M., 1984. Single-crystal elastic properties of the modified spinel (beta) phase of magnesium orthosilicate. Science 224, 749-751.

Sinogeikin, S. V., Bass, J. D., 2000. Single-crystal elasticity of pyrope and mgo to $20 \mathrm{GPa}$ by brillouin scattering in the diamond cell. Phys. Earth Planet. Int. 120, 43-62.

Stone, A. J., 1996. The theory of intermolecular forces. Oxford University Press, Oxford.

Suzuki, I., Anderson, O. L., Sumino, Y., 1983. Elastic properties of a singlecrystal forsterite $\mathrm{Mg}_{2} \mathrm{SiO}_{4}$ up to $1200 \mathrm{~K}$. Phys. Chem. Minerals 10, 38-64.

Tang, K. T., Toennies, J. P., 1984. An improved simple model for the van der Waals potential based on universal damping functions for the dispersion coefficients. J. Chem. Phys. 80, 3726-3741.

Tangney, P., Scandolo, S., 2002. An ab initio parametrized interatomic force field for silica. J. Chem. Phys. 117, 8898-8904.

Tsuneyuki, S., Tsukada, M., Aoki, H., Matsui, Y., 1988. First-principles interatomic potential of silica applied to molecular dynamics. Phys. Rev. Lett. 61, 869-872. 
van Beest, B. W. H., Kramer, G. J., van Santen, R. A., 1990. Force fields of silicas and aluminophosphates based on ab initio calculations. Phys. Rev. Lett. 64, 1955-1958.

Vanderbilt, D., 1990. Soft self-consistent pseudopotentials in a generalized eigenvalue formalism. Phys. Rev. B 41, 7892-7895.

Verma, R. K., 1960. Elasticity of some high-density crystals. J. Geophys. Res. 65, 757-766.

Weidner, D. J., Sawamoto, H., Sasaki, S., Kumazawa, M., 1984. Single-crystal elastic properties of the spinel phase of $\mathrm{Mg}_{2} \mathrm{SiO}_{4}$. J. Geophys. Res. 89, 78527860 .

Wentzcovitch, R. M., Karki, B. B., Cococcioni, M., de Gironcoli, S., 2004. Thermoelastic properties of $\mathrm{MgSiO}_{3}$-perovskite: Insights on the nature of the Earth's lower mantle. Phys. Rev. Lett. 92, 018501.

Wilson, M., Exner, M., Huang, Y.-M., Finnis, M. W., 1996a. Transferable model for the atomistic simulation of $\mathrm{Al}_{2} \mathrm{O}_{3}$. Phys. Rev. B 54 (22), 1568315689.

Wilson, M., Jahn, S., Madden, P. A., 2004. The construction and application of a fully flexible computer simulation model for lithium oxide. J. Phys.: Condens. Matter 16, S2795-S2810.

Wilson, M., Madden, P. A., Costa-Cabral, B. J., 1996b. Quadrupole polarization in simulations of ionic systems: Application to $\mathrm{AgCl}$. J. Phys. Chem. 100, 1227-1237.

Winter, J. K., Ghose, S., 1979. Thermal expansion and high temperature crystal chemistry of the $\mathrm{Al}_{2} \mathrm{SiO}_{5}$ polymorphs. Am. Mineral. 64, 573-586.

Zhang, F., Oganov, A. R., 2006. Mechanism of $\mathrm{Al}^{3+}$ incorporation in $\mathrm{MgSiO}_{3}$ post-perovskite at high pressures. Earth Planet. Sci. Lett. 248, 54-61. 


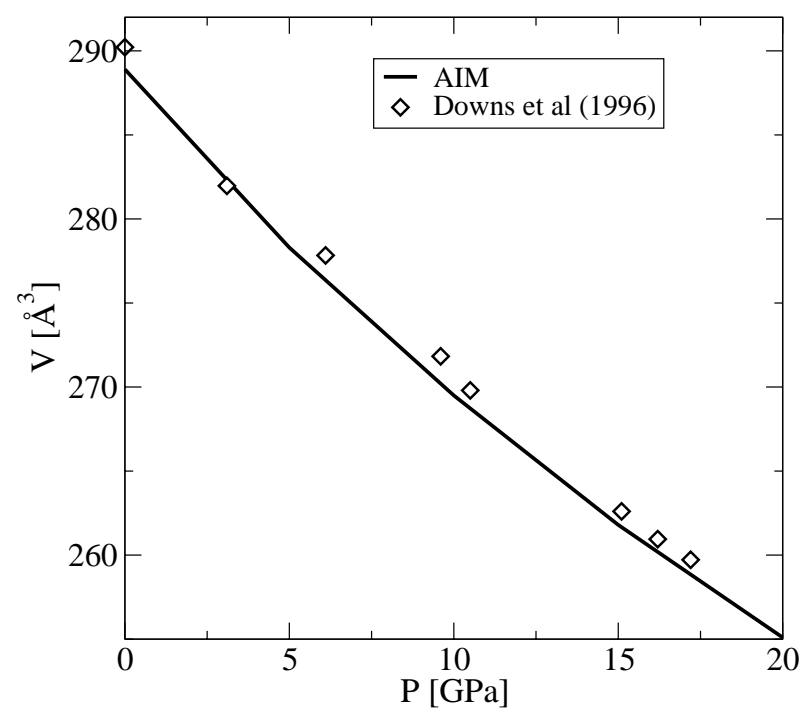

Fig. 1. Room temperature volume compression of forsterite as a function of pressure. Symbols represent experimental results from Downs et al. (1996), lines are predictions of the AIM.

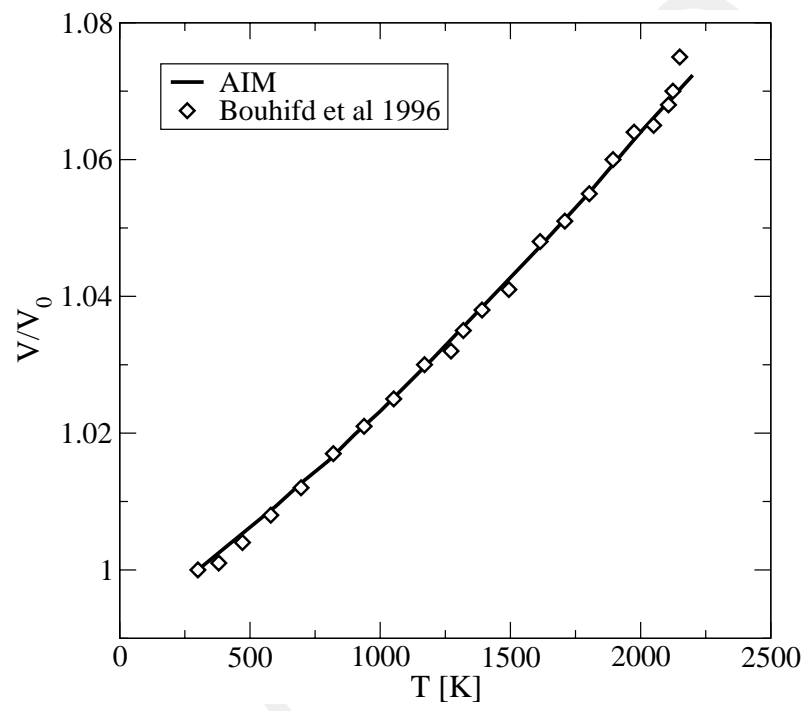

Fig. 2. Thermal expansion of forsterite at ambient pressure relative to the respective volume at room temperature. Symbols represent experimental results from Bouhifd et al. (1996), lines are predictions of the AIM. 


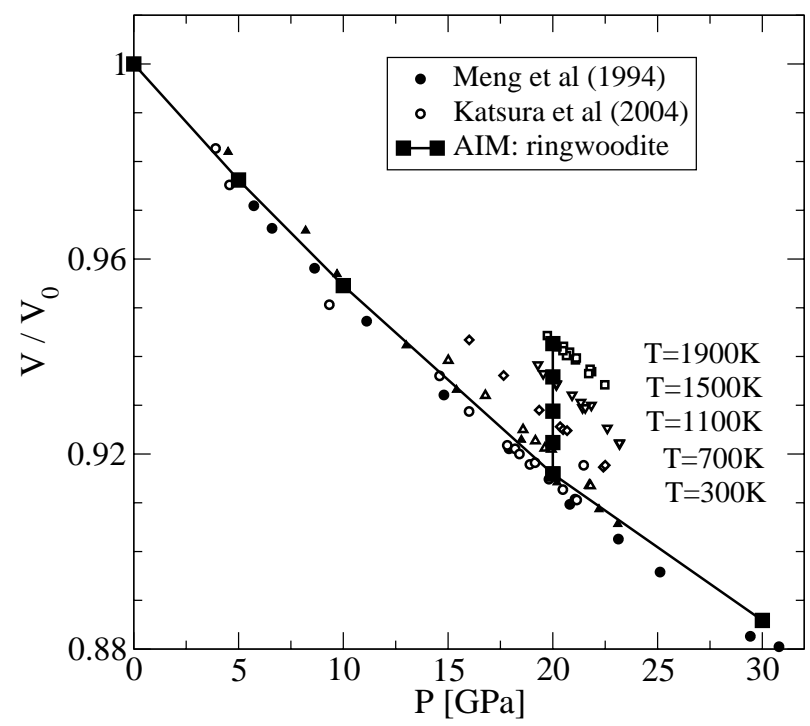

Fig. 3. Relative change of the cell volume of ringwoodite with pressure and temperature. Experimental data are represented by small filled symbols (Meng et al., 1994) and open symbols (Katsura et al., 2004), different temperatures by different symbols (circle $-300 \mathrm{~K}$, triangle up $-700 \mathrm{~K}$, diamond - $1100 \mathrm{~K}$, triangle down $1500 \mathrm{~K}$, square $-1900 \mathrm{~K}$ ). AIM predictions are shown as an isotherm at $300 \mathrm{~K}$ and an isobar at $20 \mathrm{GPa}$ (lines with large squares).

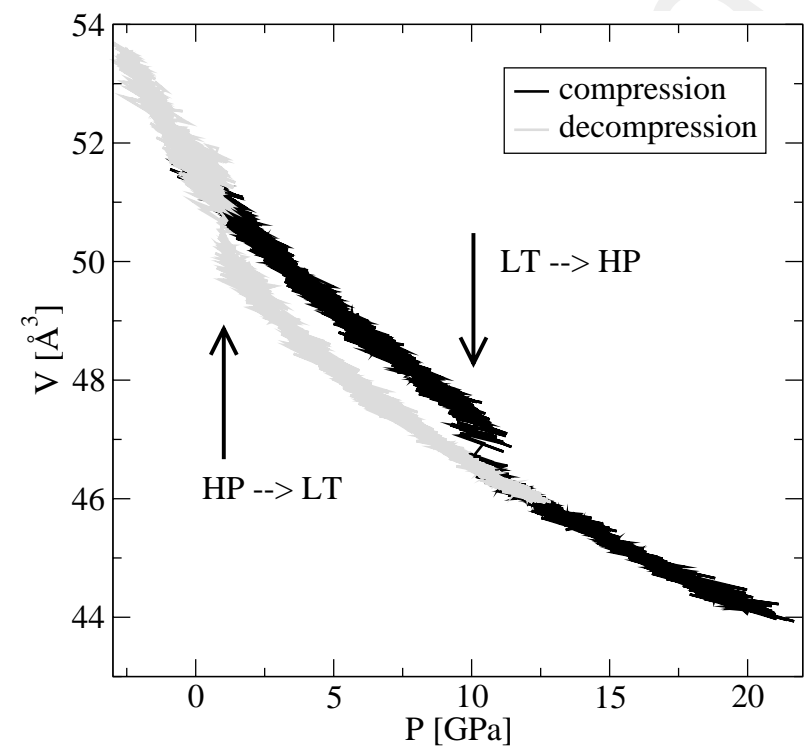

Fig. 4. Change of cell volume across the low-temperature to high-pressure clinoenstatite transition obtained from molecular dynamics simulation at constant $T=300 K$. 


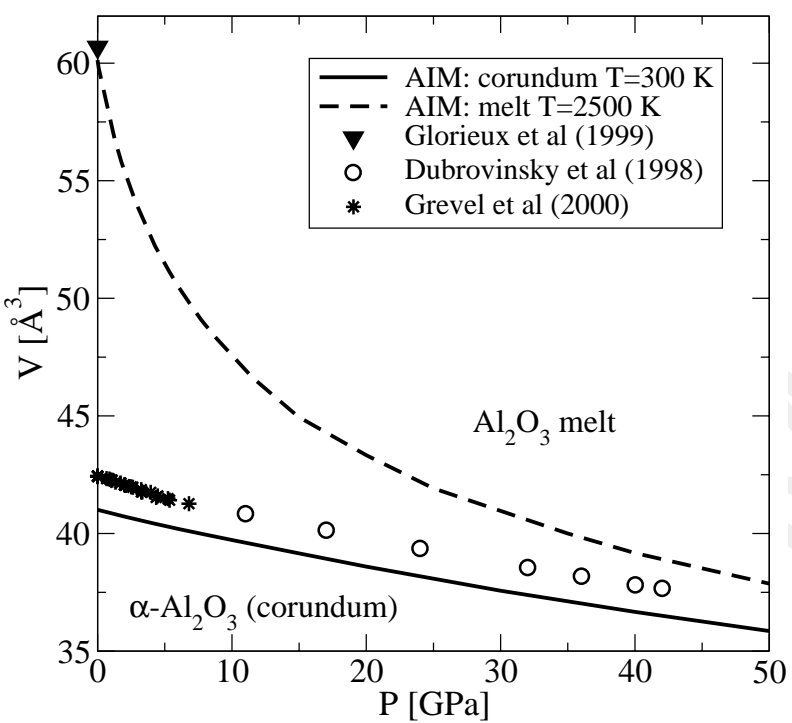

Fig. 5. Volume compression of corundum, $\alpha-\mathrm{Al}_{2} \mathrm{O}_{3}$, at $T=300 \mathrm{~K}$ and $\mathrm{Al}_{2} \mathrm{O}_{3}$ melt at $T=2500 \mathrm{~K}$ as a function of pressure. Symbol refer to experimental data (Glorieux et al., 1999; Dubrovinsky et al., 1998; Grevel et al., 2000), lines are predictions of the AIM. 
Table 1

\begin{tabular}{|c|c|c|c|c|c|}
\hline & O-O & Ca-O & Mg-O & Al-O & Si-O \\
\hline$A^{i j}$ & 1068.0 & 40.168 & 41.439 & 18.149 & 43.277 \\
$a^{i j}$ & 2.6658 & 1.5029 & 1.6588 & 1.4101 & 1.5418 \\
$B^{i j}$ & & 50532. & 59375. & 51319. & 43962. \\
$b^{i j}$ & & 3.5070 & 3.9114 & 3.8406 & 3.9812 \\
$C^{i j}$ & & 6283.5 & 6283.5 & 6283.5 & 6283.5 \\
$c^{i j}$ & & 4.2435 & 4.2435 & 4.2435 & 4.2435 \\
\hline$b_{D}^{i j}$ & & 2.0261 & 2.2148 & 2.2886 & 2.1250 \\
$c_{D}^{i j}$ & & 3.9994 & 2.8280 & 2.3836 & 1.5933 \\
$b_{Q}^{i j}$ & & 1.5297 & 1.9300 & 2.1318 & 1.9566 \\
$c_{Q}^{i j}$ & & 1.6301 & 1.3317 & 1.2508 & 1.0592 \\
\hline$C_{6}^{i j}$ & 44.372 & 2.1793 & 2.1793 & 2.1793 & 2.1793 \\
$C_{8}^{i j}$ & 853.29 & 25.305 & 25.305 & 25.305 & 25.305 \\
$b_{d i s p}^{i j}$ & 1.4385 & 2.2057 & 2.2057 & 2.2057 & 2.2057 \\
\hline \hline$D$ & 0.49566 & & $\beta$ & 1.2325 & \\
$\zeta$ & 0.89219 & & $\eta$ & 4.3646 & \\
$\alpha$ & 8.7671 & & $C$ & 11.5124 & \\
\hline
\end{tabular}

Parameters in the repulsive and polarization parts of the potential. All values are in atomic units. 
Table 2

\begin{tabular}{c|c|c|c|c} 
& Exp & Matsui & Blanchard & AIM \\
\hline$a[\AA]$ & 4.75 & 4.76 & 4.85 & 4.73 \\
$b[\AA]$ & 10.19 & 10.20 & 10.26 & 10.19 \\
$c[\AA]$ & 5.98 & 5.99 & 5.96 & 5.95 \\
\hline$c_{11}$ & 329 & 325 & 282 & 326 \\
$c_{22}$ & 200 & 203 & 160 & 188 \\
$c_{33}$ & 236 & 251 & 231 & 232 \\
$c_{44}$ & 67 & 64 & 290 & 62 \\
$c_{55}$ & 81 & 78 & 80 & 78 \\
$c_{66}$ & 81 & 83 & 75 & 82 \\
$c_{12}$ & 67 & 65 & 55 & 84 \\
$c_{13}$ & 68 & 69 & 46 & 82 \\
$c_{23}$ & 73 & 65 & 52 & 80 \\
\hline
\end{tabular}

Lattice parameters $[\AA]$ and elastic constants $[G P a]$ of forsterite at ambient conditions ( $T=0$ for the simulations). AIM predictions are compared to experimental data taken from Fujino et al. (1981) and Suzuki et al. (1983), and to the breathing shell models of Matsui (1999) and Blanchard et al. (2005). 
Table 3

\begin{tabular}{c|c|c|c|c} 
& Exp & Matsui & Blanchard & AIM \\
\hline$a[\AA]$ & 5.70 & 5.70 & 5.71 & 5.64 \\
$b[\AA]$ & 11.44 & 11.46 & 11.40 & 11.34 \\
$c[\AA]$ & 8.26 & 8.22 & 8.36 & 8.24 \\
\hline$c_{11}$ & 360 & 351 & 353 & 367 \\
$c_{22}$ & 383 & 355 & 383 & 367 \\
$c_{33}$ & 273 & 293 & 219 & 262 \\
$c_{44}$ & 112 & 104 & 101 & 95 \\
$c_{55}$ & 118 & 107 & 98 & 110 \\
$c_{66}$ & 98 & 101 & 96 & 104 \\
$c_{12}$ & 75 & 80 & 67 & 82 \\
$c_{13}$ & 110 & 92 & 82 & 103 \\
$c_{23}$ & 105 & 101 & 92 & 107 \\
\hline
\end{tabular}

Lattice parameters $[\AA]$ and elastic constants $[G P a]$ of wadsleyite at ambient conditions ( $T=0$ for the simulations). AIM predictions are compared to experimental data taken from Horiuchi and Sawamoto (1981) and Sawamoto et al. (1984), and to the breathing shell models of Matsui (1999) and Blanchard et al. (2005).

Table 4

\begin{tabular}{c|c|c|c|c} 
& Exp & Matsui & Blanchard & AIM \\
\hline$a[\AA]$ & 8.07 & 8.07 & 8.07 & 7.98 \\
\hline$c_{11}$ & 327 & 342 & 327 & 357 \\
$c_{44}$ & 126 & 127 & 126 & 130 \\
$c_{12}$ & 112 & 101 & 111 & 128 \\
\hline
\end{tabular}

Lattice parameter $[\AA]$ and elastic constants $[G P a]$ of ringwoodite at ambient conditions ( $T=0$ for the simulations). Experimental data are from Sasaki et al. (1982) and Weidner et al. (1984). Also listed are results of the models by Matsui (1999) and Blanchard et al. (2005). 
Table 5

\begin{tabular}{c|c|c||c|c} 
& \multicolumn{2}{|c||}{ pyrope } & \multicolumn{2}{c}{ grossular } \\
\hline & Exp & AIM & Exp & AIM \\
\hline$a[\AA]$ & 11.45 & 11.30 & 11.85 & 11.67 \\
\hline$c_{11}$ & 296 & 332 & 322 & 357 \\
$c_{44}$ & 92 & 103 & 105 & 116 \\
$c_{12}$ & 111 & 130 & 91 & 104 \\
\hline
\end{tabular}

Lattice parameter $[\AA]$ and elastic constants $[G P a]$ of pyrope (left) and grossular (right). Experimental data are taken from Armbruster et al. (1992); O'Neill et al. (1991) (pyrope), and Novak and Gibbs (1971); Bass (1989) (grossular).

Table 6

\begin{tabular}{c|c|c||c|c||c|c}
\multicolumn{1}{l|}{} & \multicolumn{2}{c||}{ corundum } & \multicolumn{2}{c||}{ andalusite } & \multicolumn{2}{c}{$\alpha$-quartz } \\
\hline & Exp & AIM & Exp & AIM & Exp & AIM \\
\hline$a[\AA]$ & 4.759 & 4.699 & 7.80 & 7.66 & 4.91 & 4.79 \\
$b[\AA]$ & & & 7.90 & 7.77 & & \\
$c[\AA]$ & 12.991 & 12.888 & 5.56 & 5.47 & 5.40 & 5.20 \\
\hline$c_{11}$ & 495 & 509 & 233 & 210 & 87 & 99 \\
$c_{22}$ & & & 289 & 262 & & \\
$c_{33}$ & 497 & 539 & 380 & 367 & 106 & 110 \\
$c_{44}$ & 146 & 135 & 100 & 82 & 58 & 62 \\
$c_{55}$ & & & 88 & 76 & & \\
$c_{66}$ & & & 112 & 108 & & \\
$c_{12}$ & 160 & 218 & 98 & 96 & 7 & 22 \\
$c_{13}$ & 115 & 142 & 116 & 119 & 13 & 24 \\
$c_{14}$ & -23 & -28 & & & -18 & -9 \\
$c_{23}$ & & & 81 & 99 & & \\
\hline
\end{tabular}

Lattice parameters $[\AA]$ and elastic constants $[G P a]$ of corundum, andalusite and quartz. Experimental data are taken from Newnham and de Haan (1962) (corundum), Winter and Ghose (1979); Verma (1960) (andalusite) and LePage et al. (1980); Hearmon (1979) (quartz). 
Table 7

\begin{tabular}{c|c|c||c|c} 
& \multicolumn{2}{|c||}{$\mathrm{MgSiO}_{3}$-perovskite } & \multicolumn{2}{c}{$\mathrm{MgSiO}_{3}$-postperovskite } \\
\hline & $\mathrm{DFT}$ & $\mathrm{AIM}$ & $\mathrm{DFT}$ & AIM \\
\hline$a[\AA]$ & 4.289 & 4.320 & 2.455 & 2.474 \\
$b[\AA]$ & 4.557 & 4.544 & 8.051 & 8.040 \\
$c[\AA]$ & 6.264 & 6.253 & 6.099 & 6.077 \\
\hline$c_{11}$ & 909 & 936 & 1270 & 1456 \\
$c_{22}$ & 1160 & 1190 & 937 & 1027 \\
$c_{33}$ & 1128 & 1130 & 1264 & 1328 \\
$c_{44}$ & 363 & 382 & 291 & 306 \\
$c_{55}$ & 278 & 304 & 264 & 277 \\
$c_{66}$ & 339 & 261 & 412 & 388 \\
$c_{12}$ & 520 & 530 & 425 & 435 \\
$c_{13}$ & 410 & 460 & 329 & 437 \\
$c_{23}$ & 434 & 556 & 493 & 594 \\
\hline
\end{tabular}

Lattice parameters $[\AA]$ and elastic constants $[G P a]$ of $\mathrm{MgSiO}_{3}$ perovskite and postperovskite at $P=120 \mathrm{GPa}$ and $T=300 K$ (AIM), $T=0 K(\mathrm{DFT})$. The DFT results are taken from Iitaka et al. (2004). 